\title{
A Novel Loss-of-function Pathogenic Missense Mutation in CASK Gene Causes Mental Retardation and Microcephaly with Pontine and Cerebellar Hypoplasia
}

Sixian Wu ( $\nabla$ wusixian9911@163.com )

West China Second University Hospital, Sichuan University, https://orcid.org/0000-0001-9396-9635

Chuan Jiang

West China Second University Hospital, Sichuan University,

Jiaman Li

Sichuan University West China School of Pharmacy

Guohui Zhang

West China Second University Hospital, Sichuan University,

Ying Shen

West China Second University Hospital, Sichuan University,

Jing Wang

West China Second University Hospital, Sichuan University,

Primary research

Keywords: MICPCH, CASK, missense mutation, protein structure

Posted Date: May 5th, 2021

DOl: https://doi.org/10.21203/rs.3.rs-470544/v1

License: (c) (i) This work is licensed under a Creative Commons Attribution 4.0 International License.

Read Full License 


\section{Abstract}

Background: Mutations in the CASK gene result in a wide range of observed phenotypes in humans like FG Syndrome 4 and mental retardation. In this study, researchers investigated the case of an 11-monthold female diagnosed with mental retardation and microcephaly with pontine and cerebellar hypoplasia (MICPCH). These issues caused general developmental delays, microcephaly, and cerebellar hypoplasia.

Results: Whole-exome sequencing (WES) was used to identify a novel pathogenic missense mutation (NM_003688.3: c.638T>G) in the CASK gene in this index. Researchers further confirmed the pathogenicity of the missense mutation by bioinformatics analysis. At the same time, researchers tested its protein expression level and mRNA expression level. Strikingly, the protein expression was downregulation while the mRNA expression was not changed. By using protein structure prediction, this study found that amino acid mutation resulted in further changes in the stability of protein structures, which caused down-regulation of the protein expression and loss of the protein function.

Conclusions: In this study, researchers first reported a novel causative mechanism of MICPCH: amino acid mutations led to changes in protein structure and down-regulation of stability, which caused protein was loss of function. This study can be helpful in the genetic diagnosis of this disease.

\section{Background}

Mental retardation and microcephaly with pontine and cerebellar hypoplasia (MICPCH) is an X-linked disorder that affects females and is characterized by severe intellectual disability, microcephaly, and variable degrees of pontocerebellar hypoplasia $(1,2)$. Affected individuals have very poor psychomotor development, often without independent ambulation or speech, and axial hypotonia with or without hypertonia. Some may have sensorineural hearing loss or eye abnormalities. Dysmorphic features of those affected by this condition include overall poor growth and severe microcephaly ( -3.5 to -10 SD). This causes the development of distinct facial features like broad nasal bridge and tip, large ears, long philtrum, micrognathia, and hypertelorism $(3,4)$. It has been found that the mutations of CASK, ITPR1, MARCKS, and RELN are involved in the etiology of $\mathrm{MICPCH}(5,6)$. CASK is an excellent candidate gene for the microcephaly disproportionate pontine and cerebellar hypoplasia (MICPCH) (MIM\# 300749) since this gene functions in neuronal development. CASK mutant mice have small brains, abnormal cranial shape, and a cleft palate $(4,6-10)$. The intragenic mutations of $C A S K$ have been found in more than 50 individuals with the MICPCH phenotype $(5,11)$.

The CASK gene, a member of the MAGUK protein family, maps to Xp11.4 and encodes CASK, a multidomain scaffolding protein composed of 926 amino acids (Ensembl ID: ENST00000378163), which is located at both the postsynaptic membrane of central nervous synapses and within the nuclei of neurons. $C A S K$ has a higher expression in the fetal brain $(8,12,13)$. Due to its location on the $X$ chromosome, loss-of-function of CASK usually leads to the manifestation of MICPCH in females $(3,5)$. 
Males affected by CASK mutations usually show more severe symptoms than females. These genetic issues are usually fatal in the womb for male embryos $(7,14)$.

In the clinical screening for this study, researchers first examined the case of an 11-month-old female patient with general developmental delay, microcephaly, and cerebellar hypoplasia. After screening by high-throughput sequencing detection technology of the whole exome, it was found that the patient had a novel missense mutation in CASK gene, at the location NM_003688.3: c.638T > G, p.L213R. In this study, researchers used bioinformatics methods in order to predict the pathogenicity and harm of this missense mutation in CASK. Researchers also examined the mRNA expression as well as protein expression of the mutation and predicted the structure of the protein. This study found that there is no difference between mRNA expression; however, amino acid mutations were found to cause downstream changes in the stability of the protein's spatial structure, which causes down-regulation of protein expression and loss of protein function.

\section{Results}

\section{Clinical summary}

An unrelated natural couple brought an 11-month-old female with delayed development to the outpatient for genetic counseling (Figure 1A). The proband exhibited features of general developmental delay, jaundice, clenched hands, and microcephaly. Her parents told us the patient had problems with sleeping, slow movement, and other developmental issues. Through testing, researchers found that the patient had increased muscle tone, dystonia, and cerebellar atrophy (Figure 1B).

Axial brain magnetic resonance imaging (MRI) was performed on the patient to obtain further information. The MRI revealed decreased size of both cerebellar hemispheres, widening of sulci in both cerebral hemispheres, and widening and deepening of cerebellar sulci (Figure 1C). Researchers initially diagnosed the patient with mental retardation and microcephaly with pontine and cerebellar hypoplasia (MICPCH) depending on the specific clinical characteristics. There were no phenotypic abnormalities observed in the parents of the patient.

\section{Bioinformatic Analysis}

Researchers extracted the DNA from the patient's peripheral blood and performed WES. Results showed that the CASK gene had a missense mutation, specifically CASK: NM_003688.3: exon7: c.638T>G: p.L213R. (Figure 1D) (15).

According to the bioinformatics analysis, there was no previous report about this variation in most databases, including ExAC browser, 1000 genome project, or In-house Chinese-Control. The latest gnomAD database shows the frequency of this mutation as 0.000005520 (16) (Table 1). In addition, this variant site is highly conserved in many species according to mutation taster (Figure 2A). PhastCons and 
PhyloP were used to evaluate the scores of amino acid sequence conservation. Scores indicated that this mutation site is highly conserved (Table 1). Moreover, this mutation was predicted to be pathogenic by the following bioinformatic tools: SIFT(17), PolyPhen-2(18), and M-CAP(19) (Table 1). The above results found this mutation site to be pathogenic and well conserved.

\section{Table 1}

The information of the missense mutation in CASK gene

\begin{tabular}{|lll|}
\hline Gene & CASK & \\
\hline cDNA Mutation & NM_003688.3: c.638T >G & \\
\hline Variant Allele Frequency & ExAC Browser & 0 \\
\cline { 2 - 3 } & GnomAD & 0.000005520 \\
\cline { 2 - 3 } & 1000 Genomes Project & 0 \\
\cline { 2 - 3 } & In-house Chinese-Control & 0 \\
\hline Amino acid & PhyloP & 3.907 \\
\cline { 2 - 3 } sequence conservation & PhastCons & 1 \\
\hline Function prediction & SIFT & Deleterious \\
& PolyPhen-2 & Probably damaging \\
\cline { 2 - 3 } & M-CAP & Possibly pathogenic \\
\hline
\end{tabular}

\section{Molecular genetic analysis}

In order to further explore the molecular disease mechanism caused by the missense mutation of the CASK gene, wild-type and mutant plasmids were constructed and transferred into HEK-293T cells.

Researchers tested the mRNA expression (Figure 3B) and protein expression (Figure 3A\&C) of wild and mutant type, and found that there was no significant difference in mRNA expression between the wild type and the mutant type while compared with the wild type, the protein expression of the mutant type is down-regulated.

Finally, researchers investigated the structural pattern of the protein after the amino acid arginine (R) was substituted for leucine $(\mathrm{L})$ by PSIPRED(20-22). The results of protein structure prediction showed that the nuclear charge of the protein increased $(\Delta$ Charge $=1)$ and the stability of the protein decreased $\left(\Delta \Delta G_{\text {pred }}=1.857\right)$ after the mutation. At the same time, a random coil in the secondary structure is changed to $\beta$-sheet, which also affects its spatial structure (Figure 2B). Decreased protein stability directly leads to protein degradation, which is also the reason for the down-regulation of protein expression. The above caused the loss of protein function. 


\section{Discussion}

In this study, researchers examined the case of a female with a novel pathogenic missense mutation in CASK that is associated with MICPCH. Moreover, the significant results of decreased size of both cerebellar hemispheres, widening of sulci in both cerebral hemispheres, and widening and deepening of cerebellar sulci were observed on the results of MRI. The results of the WES sequencing show CASK gene had a missense mutation of CASK: NM_003688.3: exon7: c.638T>G: p.L213R. Although there was no significant difference found in the expression of the missense mutation in CASK by detecting the mRNA expression, the protein expression is down-regulation. By using the method of protein structure prediction, researchers found that when the leucine in the primary sequence of the protein was mutated to arginine, protein stability was reduced and secondary structure as well as spatial structure were changed. This resulted in reduced protein expression and loss of protein function.

All of the residues in the aF helix have been shown to be involved in a conserved spatial pattern. Kornev et al. (2008)(23) suggest that the residue at the position equivalent to 209 in CASK plays a critical role in the anchoring of the aH helix. This is purported to help stabilize the hydrophobic core around the aF helix. (12) Similarly, in this study, the leucine at position 213 in the amino acid sequence of the CASK protein is mutated to arginine. Since arginine is a basic amino acid and leucine is a non-polar amino acid (24), the amino acid change cause the change of maintenance of the third structure level in the protein (25). The reduction of hydrophobic force may not stabilize the hydrophobic core around the aF, which can greatly disrupt the function of $C A S K$ as a protein kinase.

Generally, the types of mutations that cause MICPCH are nonsense mutations, splice site mutations, shifting-frame mutations, and missense mutations. These types of mutations usually result in a decrease in CASK protein expression. The loss of CASK expression was associated with a more severe $\mathrm{MICPCH}$ phenotype and likely caused the reduced viability or in utero lethality. This research once again confirmed the loss of function causes severe general developmental delay, microcephaly, and cerebellar hypoplasia. And it is rare that changed protein structure and increased instability in CASK protein leads to downregulation of protein expression and loss of function. These findings extend the variety of genetic alterations causing $C A S K$ mutations. Moreover, this research is of great significance for understanding the pathogenicity of $C A S K$ point mutation. This study further reveals the key role of $C A S K$ in MICPCH development, and helps provide genetic explanations for the etiology of $C A S K$ mutation in $\mathrm{MICPCH}$ affected patients.

\section{Conclusions}

In conclusion, this research first reports that the novel pathogenic missense mutation in CASK (NM_003688.3: exon7: c.638T > G: p.L213R) in the 11-month-old female caused mental retardation and microcephaly with pontine and cerebellar hypoplasia (MICPCH). This study also determined the bioinformatic content of this mutation site and explored there was no differences in mRNA expression while protein expression was down-regulation. Finally, the researchers analyzed the structural changes of 
the protein and confirmed that the cause of the disease was the down-regulation of the protein caused by amino acid mutations that changed the stability of the protein's structure.

\section{Methods}

\section{Subjects}

Peripheral blood samples were obtained from the proband and her family after informed consent was signed by the patient and all family members. This experiment on human subjects was approved by the Ethical Review Board of West China Second University Hospital, Sichuan University. The patient's brain MRI results were evaluated by the pediatric neuroradiologist.

\section{Whole-exome sequencing}

Genomic DNA was extracted from peripheral blood leukocytes using a whole blood DNA purification kit (TIANGENE). For WES, exons were captured from $1 \mu \mathrm{g}$ genomic DNA using high-throughput sequencing detection technology for the whole exome provided by the manufacturer. The Verita Trekker ${ }^{\circledR}$ variant site detection system and Enliven ${ }^{\circledR}$ variant site annotation interpretation system independently developed by Berry Genomics were used to analyze the data. Functional annotation was performed through ANNOVAR, and data were filtered by public databases such as ExAC, 1000 Genomes Project, and GnomAD .

Candidate pathogenic variants in the family members and their flanking intronic regions of CASK in the unrelated population were validated by Sanger sequencing. PCR amplification was performed with Dyad Polymerase (Bio-Rad Laboratories). DNA sequencing of PCR products was conducted on an ABI377A DNA sequencer (Applied Biosystems). The primers for PCR are listed as follows:

\section{5' TTTCCTGGACAGCTACAGgC 3', 5' CTGgCAGTCAATTAGTGgGCA 3'}

\section{Plasmid construction and cell transfection}

The full-length cDNA of CASK was synthesized and separately cloned into pCMV-MCS-3*Flag. The CASK plasmids were synthesized and cloned by Gene Company (Shanghai, China). All mutant plasmids of CASK were generated by the Fast Mutagenesis System of TransGen Biotech Co. Ltd (Beijing, China). The constructed plasmid was transformed into $E$. coli. Then, $10 \mu \mathrm{L}$ of plasmid-containing bacterial solution and $20 \mu \mathrm{L}$ of antibiotic kana $(50 \mathrm{mg} / \mathrm{mL})$ were added to $20 \mathrm{~mL}$ of Luria-Bertani medium. The bacteria were placed on a shaker at $37^{\circ} \mathrm{C}$ overnight. After shaking was complete, researchers extracted the plasmid from E. coli.

The HEK-293T cell line was obtained from the American type culture collection (ATCC, USA). The 293T cells were cultured in 6-well cell culture plates and $100 \mathrm{~mm}$ cell culture dishes (WHB, China) with basic DMEM medium containing $10 \%$ fetal bovine serum (Gibco, USA) and $0.1 \%$ penicillin/streptomycin in a 
humidified incubator at $37^{\circ} \mathrm{C}$ with $5 \% \mathrm{CO}_{2}$. According to the experimental scheme, $C A S K$ plasmids were transfected into $293 \mathrm{~T}$ cells for $24-48 \mathrm{~h}$.

\section{Quantitative PCR}

The total RNA of the cell was extracted using TRIzol reagent (Invitrogen) and was converted to cDNA using a Revert Aid First-Strand cDNA Synthesis Kit (ThermoFisher). Quantitative PCR was performed using SYBR Premix Ex Taq II (TaKaRa) on an iCycler RT-PCR Detection System (Bio-Rad Laboratories).

The $\triangle \triangle C T$ method was used for data analysis. Each assay was performed in triplicate for each sample. The GADPH gene was used as an internal control.

The primers of human GADPH are as follows:

5' ATGTTCGTCATGggtgtgAA 3',

5' GTCTTCTGGGTGGCAGTGAT 3'

The primers of human CASK are as follows:

5' GACTTGTAGCTGGAGGACGTG 3'

5' GATATGGCTCCACTGCCTTGG 3'

\section{Western blotting}

The proteins were extracted using radioimmunoprecipitation assay (RIPA) buffer that contained a protease and phosphatase inhibitor cocktail (Roche). Twenty microgram of the denatured proteins were separated with the use of $10 \%$ SDS-polyacrylamide gels and transferred to a polyvinylidene difluoride (PVDF) membrane (Millipore) for immunoblotting analysis. After blocking with Tris-buffered saline/Tween-20 (TBST) containing $5 \%$ bovine serum albumin (BSA) for $1 \mathrm{~h}$ at room temperature, the membranes were then incubated with the corresponding primary antibodies 1:50 anti-FLAG (HPA052219; Sigma-Aldrich) and 1:2000 anti- $\beta$-actin (T7451; Sigma-Aldrich) at $4^{\circ} \mathrm{C}$. Samples were incubated overnight. The binding of the primary antibodies was visualized using horseradish peroxidase-conjugated goat antirabbit or antimouse IgG (1:10,000, ZSGB-BIO, China). The signal intensities were measured using ECL (1305702; Millipore Corporation, Billerica, USA) and image analysis software (ImageJ, NIH).

\section{Immunofluorescence}

The cells transferred into the plasmid were cultured in a glass-bottom dish. Then the cell samples were fixed onto slides with using $4 \%$ paraformaldehyde for $10 \mathrm{~min}$, the followed by slides were washed by with 
PBS. The slides were permeabilisedzed with $0.3 \%$ Triton X-100 and blocked with $5 \%$ BSA in PBS. Next, the slides were then incubated with the corresponding primary antibodies (anti-FLAG 1:50) overnight at $4{ }^{\circ} \mathrm{C}$. Second on the following day, the slides were washed by PBS, then incubated with Alexa Fluor 488 (1:500) (A21206; Thermo Fisher) labelled secondary antibodies for $1 \mathrm{~h}$ at room temperature, and then counterstained with 4,6-diamidino-2-phenylindole (DAPI,) (Sigma-Aldrich) to label the nuclei. Images were obtained using a laser scanning confocal microscope, 60X (Olympus).

\section{Abbreviations}

MICPCH: Mental retardation and microcephaly with pontine and cerebellar hypoplasia

FGS4: FG Syndrome 4

MRI: Magnetic resonance imaging

WES: Whole-exome sequencing

\section{Declarations}

\section{Acknowledgements}

We thank the patient who provided samples for this research.

\section{Authors' contributions}

W.X., J.C., and S.Y. designed the experiments and conceptualized the study. W.X. and L.J. analyzed the data. W.X., Z.G., and S.Y. performed the experiments and wrote the manuscript. W.J. and S.Y. supervised and approved the final draft of the document. All authors have read and agreed to the published version of the manuscript.

\section{Funding}

Not applicable.

\section{Availability of data and materials}

The data that support the findings of this study are available from the corresponding author upon reasonable request.

\section{Ethics approval and consent to participate}


Experiment on human subjects was approved by the Ethical Review Board of West China Second University Hospital, Sichuan University. Informed consent for participation to this study was obtained from all individuals.

\section{Consent for publication}

Publication of data was informed consent to all individuals involved in this study.

\section{Competing interests}

The authors declare no conflicts of interest.

\section{References}

1. LaConte LEW, Chavan V, Elias AF, Hudson C, Schwanke C, Styren K, et al. Two microcephalyassociated novel missense mutations in CASK specifically disrupt the CASK-neurexin interaction. Human Genetics. 2018;137(3):231-46.

2. Cristofoli F, Devriendt K, Davis EE, Van Esch H, Vermeesch JR. Novel CASK mutations in cases with syndromic microcephaly. Hum Mutat. 2018;39(7):993-1001.

3. Moog U, Kutsche K, Kortum F, Chilian B, Bierhals T, Apeshiotis N, et al. Phenotypic spectrum associated with CASK loss-of-function mutations. J Med Genet. 2011;48(11):741-51.

4. Saitsu H, Kato M, Osaka H, Moriyama N, Horita H, Nishiyama K, et al. CASK aberrations in male patients with Ohtahara syndrome and cerebellar hypoplasia. Epilepsia. 2012;53(8):1441-9.

5. Hayashi S, Uehara DT, Tanimoto K, Mizuno S, Chinen Y, Fukumura S, et al. Comprehensive investigation of CASK mutations and other genetic etiologies in 41 patients with intellectual disability and microcephaly with pontine and cerebellar hypoplasia (MICPCH). PLoS One. 2017;12(8):e0181791.

6. Hayashi S, Okamoto N, Chinen Y, Takanashi J, Makita Y, Hata A, et al. Novel intragenic duplications and mutations of CASK in patients with mental retardation and microcephaly with pontine and cerebellar hypoplasia (MICPCH). Hum Genet. 2012;131(1):99-110.

7. Najm J, Horn D, Wimplinger I, Golden JA, Chizhikov VV, Sudi J, et al. Mutations of CASK cause an Xlinked brain malformation phenotype with microcephaly and hypoplasia of the brainstem and cerebellum. Nat Genet. 2008;40(9):1065-7.

8. Froyen G, Van Esch H, Bauters M, Hollanders K, Frints SG, Vermeesch JR, et al. Detection of genomic copy number changes in patients with idiopathic mental retardation by high-resolution X-array-CGH: important role for increased gene dosage of XLMR genes. Hum Mutat. 2007;28(10):1034-42.

9. Becker M, Mastropasqua F, Reising JP, Maier S, Ho ML, Rabkina I, et al. Presynaptic dysfunction in CASK-related neurodevelopmental disorders. Translational psychiatry. 2020;10(1):312. 
10. Atasoy D, Schoch S, Ho A, Nadasy KA, Liu X, Zhang W, et al. Deletion of CASK in mice is lethal and impairs synaptic function. Proceedings of the National Academy of Sciences of the United States of America. 2007;104(7):2525-30.

11. Burglen L, Chantot-Bastaraud S, Garel C, Milh M, Touraine R, Zanni G, et al. Spectrum of pontocerebellar hypoplasia in 13 girls and boys with CASK mutations: confirmation of a recognizable phenotype and first description of a male mosaic patient. Orphanet J Rare Dis. 2012;7:18.

12. LaConte LEW, Chavan V, DeLuca S, Rubin K, Malc J, Berry S, et al. An N-terminal heterozygous missense CASK mutation is associated with microcephaly and bilateral retinal dystrophy plus optic nerve atrophy. Am J Med Genet A. 2019;179(1):94-103.

13. Cohen AR, Woods DF, Marfatia SM, Walther Z, Chishti AH, Anderson JM. Human CASK/LIN-2 binds syndecan-2 and protein 4.1 and localizes to the basolateral membrane of epithelial cells. J Cell Biol. 1998;142(1):129-38.

14. Moog U, Bierhals T, Brand K, Bautsch J, Biskup S, Brune T, et al. Phenotypic and molecular insights into CASK-related disorders in males. Orphanet J Rare Dis. 2015;10:44.

15. Richards S, Aziz N, Bale S, Bick D, Das S, Gastier-Foster J, et al. Standards and guidelines for the interpretation of sequence variants: a joint consensus recommendation of the American College of Medical Genetics and Genomics and the Association for Molecular Pathology. Genetics in medicine : official journal of the American College of Medical Genetics. 2015;17(5):405-24.

16. Kearney HM, Thorland EC, Brown KK, Quintero-Rivera F, South ST. American College of Medical Genetics standards and guidelines for interpretation and reporting of postnatal constitutional copy number variants. Genetics in medicine : official journal of the American College of Medical Genetics. 2011;13(7):680-5.

17. Kumar P, Henikoff S, Ng PC. Predicting the effects of coding non-synonymous variants on protein function using the SIFT algorithm. Nature protocols. 2009;4(7):1073-81.

18. Adzhubei IA, Schmidt S, Peshkin L, Ramensky VE, Gerasimova A, Bork P, et al. A method and server for predicting damaging missense mutations. Nature methods. 2010;7(4):248-9.

19. Jagadeesh KA, Wenger AM, Berger MJ, Guturu H, Stenson PD, Cooper DN, et al. M-CAP eliminates a majority of variants of uncertain significance in clinical exomes at high sensitivity. Nat Genet. 2016;48(12):1581-6.

20. McGuffin LJ, Bryson K, Jones DT. The PSIPRED protein structure prediction server. Bioinformatics (Oxford, England). 2000;16(4):404-5.

21. Buchan DWA, Jones DT. The PSIPRED Protein Analysis Workbench: 20 years on. Nucleic acids research. 2019;47(W1):W402-w7.

22. Buchan DW, Minneci F, Nugent TC, Bryson K, Jones DT. Scalable web services for the PSIPRED Protein Analysis Workbench. Nucleic acids research. 2013;41(Web Server issue):W349-57.

23. Kornev AP, Taylor SS, Ten Eyck LF. A helix scaffold for the assembly of active protein kinases. Proceedings of the National Academy of Sciences of the United States of America. 
2008;105(38):14377-82.

24. Grantham R. Amino acid difference formula to help explain protein evolution. Science (New York, NY). 1974;185(4154):862-4.

25. Vitkup D, Sander C, Church GM. The amino-acid mutational spectrum of human genetic disease. Genome biology. 2003;4(11):R72.

\section{Figures}


A

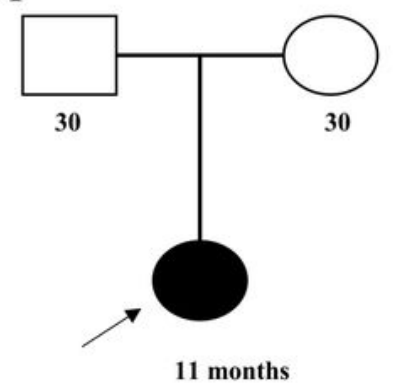

B
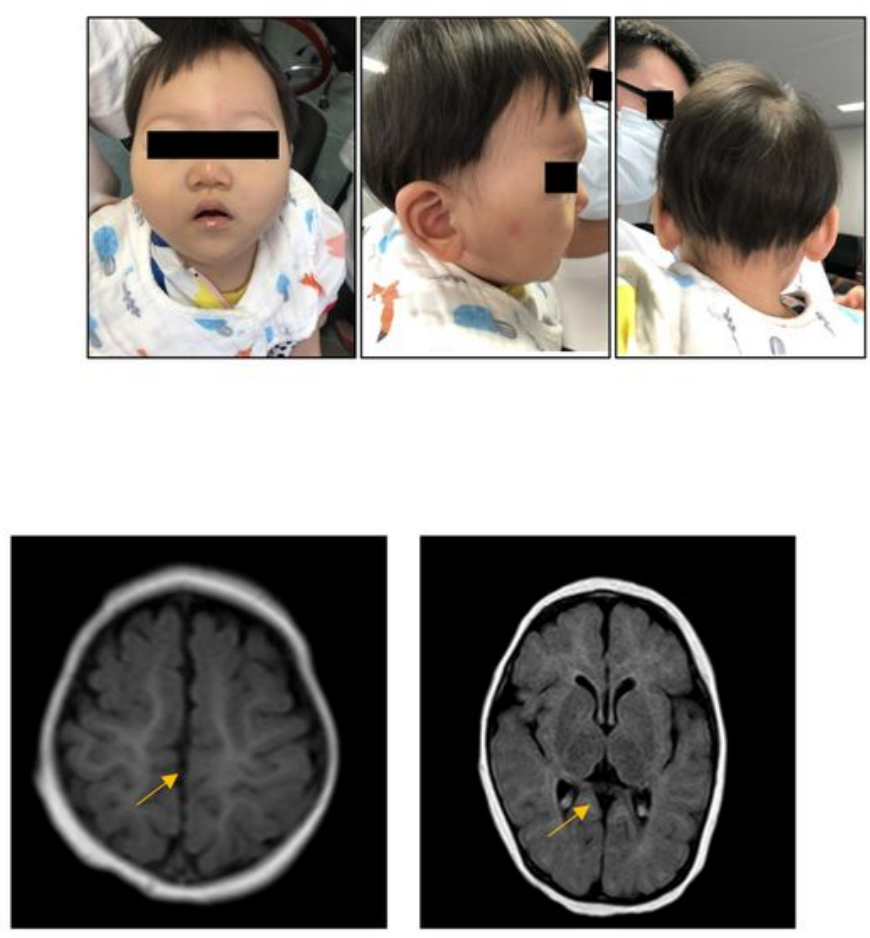

D Reference

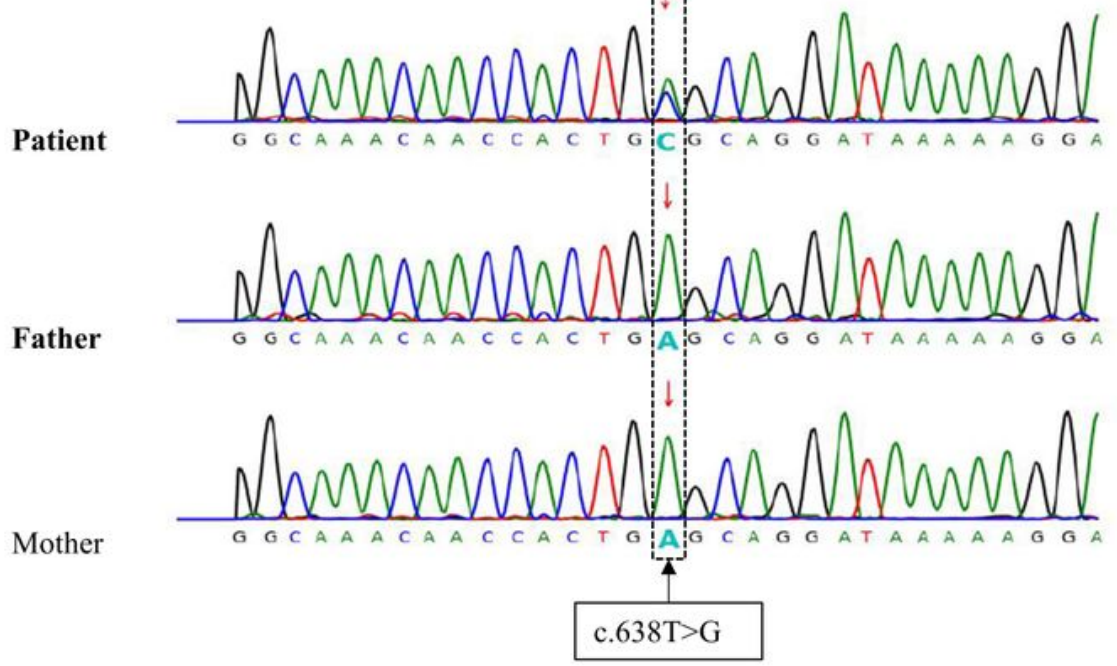

\section{Figure 1}

Clinical summary for the patient family. (A) Family pedigree. An unrelated natural couple who gave birth to the affected female. Black arrow denotes the proband. (B) and (C) The patient's axial brain MRI, which indicates the cerebral fissure widens and cerebellum shrinks. (D) PCR sequencing confirmed CASK: NM_003688.3: exon7: c.638T>G: p.L213R mutation in this family. 
A

\begin{tabular}{|c|c|c|}
\hline Species & AA & Sequence \\
\hline Human & 213 & V W G C G V I L F I L L G C L P F Y G T K E \\
\hline Ptroglodytes & 192 & VW G C G V I L F I L L G C L P F Y G T K E \\
\hline Mmulatta & 213 & V W G C G V I L F I L L G C L P F Y G T K E \\
\hline Fcatus & 193 & VW G C G V I L F I L L G C L P F Y G T K E \\
\hline Mmusculus & 213 & VW G C G V I L F I L L G C L P F Y G T K E \\
\hline Ggallus & 213 & VW G C G V I L F I L L G C L P F Y G T K E \\
\hline Trubripes & 213 & VW G C G V I L F I L L G C L P F Y G T K E \\
\hline Drerio & 200 & VW G C G V I L F I L L G C L PF Y G T K E \\
\hline
\end{tabular}

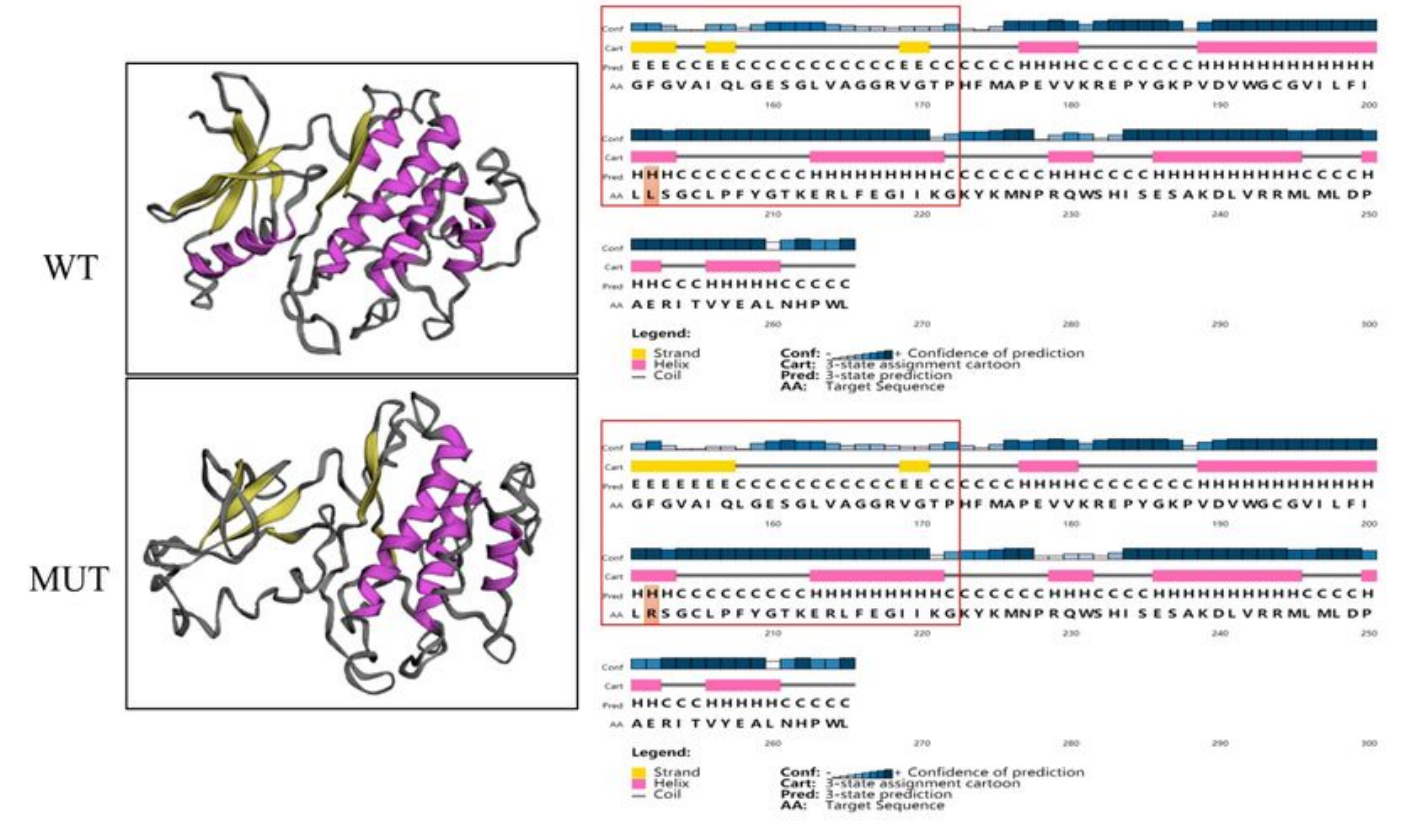

\section{Figure 2}

Bioinformatic analysis of the variants in CASK. (A) Multiple sequence alignment of the CASK protein for different species. (Black arrow denotes the position of the variant) (c.638T>G: p.L213R). (B) The secondary and spatial structure prediction of the WT and MUT protein. 
A

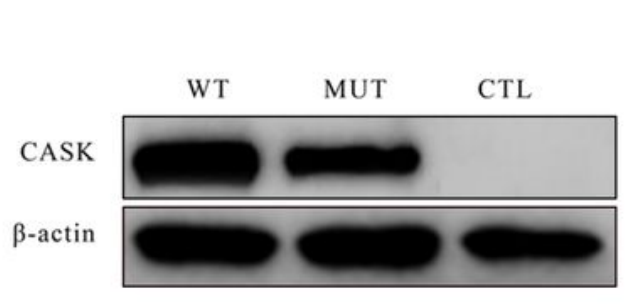

B

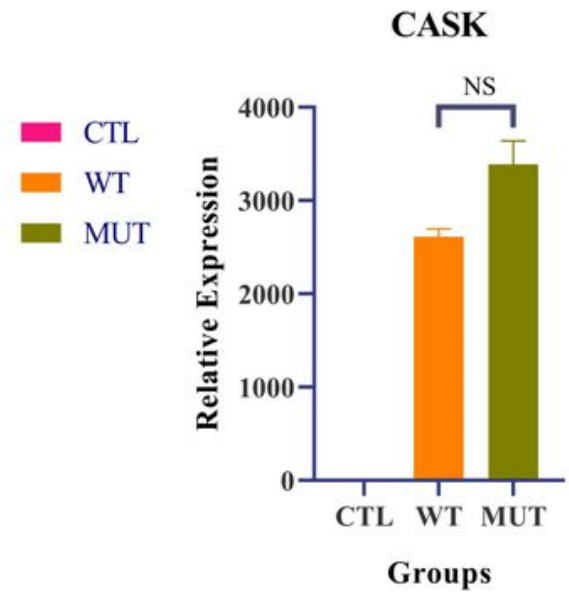

$\mathrm{C}$

CTL
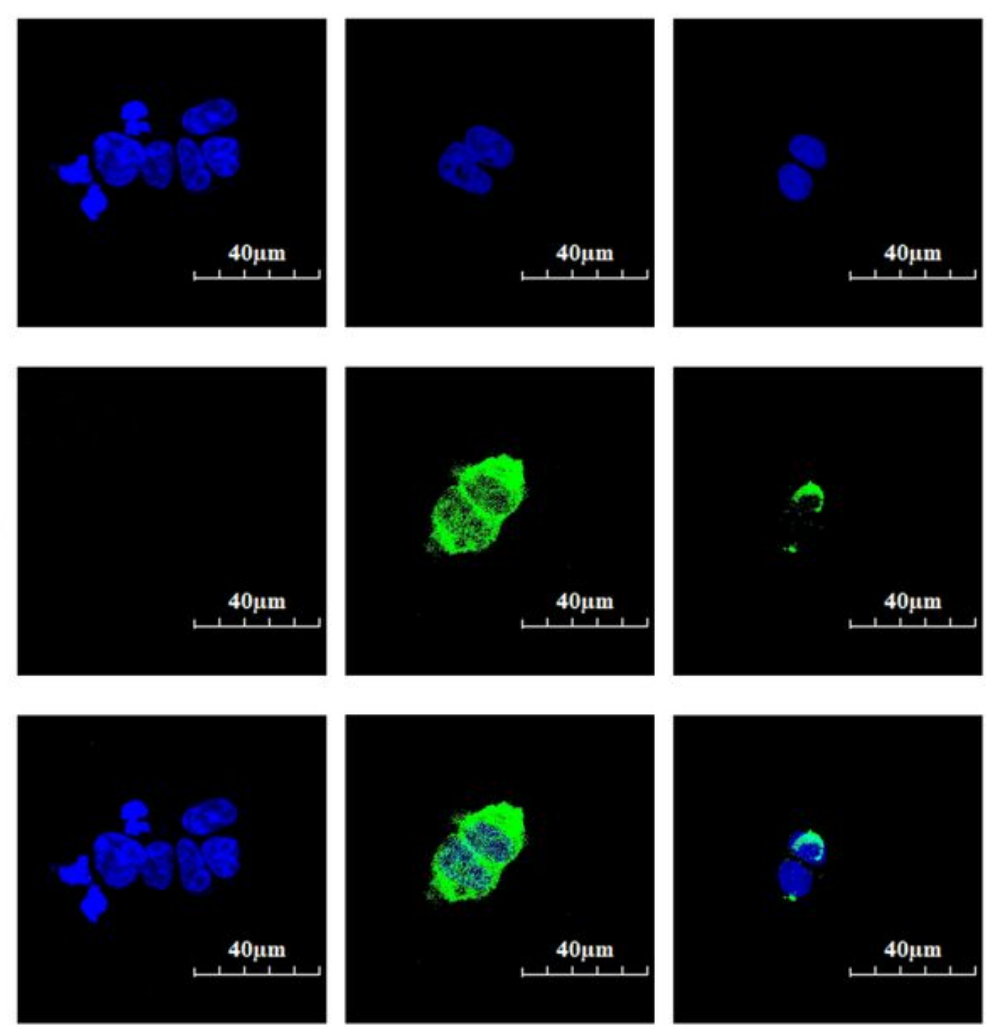

\section{Figure 3}

Molecular genetic analysis of the variants in CASK. (A) and (C) Western blotting and immunofluorescence revealed down-regulation of protein expression after mutation. (B) qPCR revealed there is no significant difference between the WT and the MUT. 